\title{
Analysis of Localized Actors' Behaviors in Service Ecosystem Simulation
}

\author{
Satoru Fujita \\ Hosei University \\ fujita_s@hosei.ac.jp
}

\begin{abstract}
This paper analyzes social phenomena resulted in service-dominant $(S-D)$ logic from a quantitative viewpoint with agent-based simulation. An agent plays a role of an actor in $S-D$ logic, who integrates resources, collaborates with other actors, and exchanges service to serve values to beneficiaries. In the simulation, actors started as generic actors, became specialized into a single type of skill, and configured various patterns of institutional arrangements and service ecosystems. Especially, when the simulation constrained a trip distance for one-time exchange, and made the actors' life levels variable corresponding to the obtained values, many small local structures of actors appeared in the ecosystems. Moreover, we also changed the land model into that with multiple fertile points, and then observed small satellite villages appeared apart from the central villages. These observations stand for that local institutions were endogenously established under contextual constraints from a $S$-D logic viewpoint.
\end{abstract}

\section{Introduction}

Service-dominant (S-D) logic has been proposed as a theoretical foundation for understanding economic activities from a service-for-service perspective [8]. In this logic, service is defined as mutual interaction in a value cocreation process among general actors. Actors integrate their operand and operant resources by themselves or with the others' resources to create values, and sometimes exchange their service with each other to complement their own value cocreation processes. A lot of papers and books have been published to explain and discuss the related issues on S-D logic [7][8][9][13], but only a few analyzed social phenomena resulted in S-D logic from a quantitative viewpoint.

Our previous work examined a value cocreation process with agent-based simulation [4][5]. In those simulations, an agent behaves as a general actor with several service resources and collaborates with each other to create values and complement his/her capability, as in the basic definitions of S-D logic. Then, we observed that agents started as self-contained actors who achieved all tasks necessary for their lives by themselves, endogenously constructed groups, organization and institutional arrangements, and finally figured out service ecosystems in their society. Their growing stages were not monotonic, and several phase transitions were repeated [5], as dynamics of ecological systems described by Holling [6].

The results in the former papers were meaningful as a first step, but the social phenomena observed in the simulations were rather simple and a whole society was governed by a single context. S-D logic defines that value is always uniquely and phenomenologically determined by the beneficiaries, and value cocreation is coordinated through actor-generated institutions and institutional arrangements [12]. If the generated society follows this logic, various values should be created and local institutions should be generated in the simulation. Therefore, a total structure of the society should become more complex. 'Through complex interactions among multiple actors, shaped by various institutional arrangements, value is continually co-created and codestroyed and cannot be determined ex-ante' [14]. This paper examines which factors make a society establish such various local structures in simulation. Especially, we focus on imposing trip restriction for exchange, variable life levels and scattered fertile regions.

Through the simulation, actors were located in a two-dimensional map, and constrained their capabilities of resource integration and service exchange with a distance between actors. An agent easily collaborated with another agent close to each other, and found exchange partners within a distance. Therefore, physical location was the first thing to be considered in this view. We also introduce an alternative viewpoint to interpret our simulation; an actor location is not a physical location, but a conceptual location among human relations. For example, the internet connects people far from each other with a tightly coupled communication line, ignoring any geographical distance. If two actors have 
the common interests, they can work together to cocreate values for them through the internet. In such a case, a distance between actors is not a geographical one, but a relational one. Such reinterpretation of the map and distance in the simulation points out another explanation in actors' behaviors. This paper describes behaviors observed in local community from this viewpoint.

\section{Service-Dominant Logic and Simulation}

\subsection{Service-Dominant Logic}

Vargo and Lusch introduced S-D logic in their paper [9], in which they proposed an inverted interpretation of economic activities traditionally explained from a goods-dominant viewpoint [7][10][12]. The basic concepts in S-D logic are interdependent. Actors are fundamentally not different from each other, but vary in their levels of operant resources (e.g., skills and abilities). Service consists of the application of multiple resources and is gathered and integrated by actors. Actors exchange service to satisfy their own requirements for living.

For service exchange, actors depend on rules, called institutions [11][12]. Some of these institutions are formalized (e.g. laws) and thus appear to be externally given, while others exist informally and endogenously emerge. All of them are, however, selectively applied. These service activities, over time, stabilize value cocreating practices, resulting in discernible patterns. All emerge from actors' individual activities. In S-D logic, an interdependent structure among actors is called a service ecosystem, conceptualized in terms of reciprocally serviceproviding actors, coordinated by institutions. Value cocreation processes are recursive and change institutions and ecosystems dynamically. Such dynamism is embedded in value cocreation and service ecosystems -- relatively self-contained, self-adjusting systems of resource-integrating actors connected by shared institutional arrangements and mutual value creation through service exchange [11].

\subsection{Service Ecosystem Simulation}

We simulated service ecosystems with an agent based model in our previous work [4][5]. In that model, an agent works as a roll of an actor that has operant resources, such as catching fish, growing wheat, exchanging and collaboration. An actor's essential value is defined as keeping alive as long as possible, and for achieving this purpose, the actor needs to ingest certain amounts of both protein and

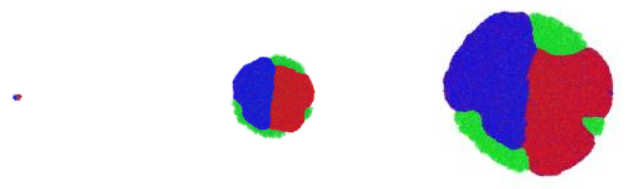
(1) Stage1
(2) Stage2
(3) Stage3

Fig. 1 Typical pattern of growing villages

carbohydrate. To obtain the protein and carbohydrate, it needs to catch fish, grow wheat and cooperate with the other actors. If actors collaborate on catching fish with others, a total amount of fish becomes more than the sum of the individual amounts obtained by their separate tasks. If an actor has a sufficient amount of fish, but lack of wheat, it must explore to find an exchange partner who has opposite resources and exchange service values. The exchange also requires that the actor has a certain level of the operant resource for exchange and allocates a certain amount of time for exchange. We define the exchange skill affects a distance the actor can travel for exchange. If an actor has higher skill for exchange, and spends a sufficient amount of time for exchange, it can travel a distance enough to visit and connect many actors. On the other hand, if an actor exclusively concentrates on catching fish and cannot allocate a time enough to exchange its service, the actor cannot exchange any by itself, and needs to ask a marketer who is specialized for exchange service to help it to exchange its service.

A world map is given as a cell structure, and each cell involves zero or one actor. The shape of the world map is a torus, and the map size is $800 \times 800$ or $1000 \times 1000$ cells. An actor who obtains 1.0 fish and 1.0 wheat in a period can survive on the land and generate a child actor every five periods. There are several initial settings, but, in most cases, the center circle of the land is fertile and other cells in the land are poor. As an initial state, actors are allocated at the fertile center circle, and then expand their residential area to the poor land that are located out of the center circle.

The simulation was done with various conditions, such as a fertile level of the land, the number of collaboration partners, a search mechanism for exchange partners, and so on. The following is a typical case we observed in the simulation, as shown in Fig. 1, where actors are colored by their skill levels; blue indicates higher skill for fishing, red for farming, and green for marketing. When actors started expanding their residential area out of the center circle, they were specialized into fishing or farming skills and made collaborative groups with the actors who were specialized into the same skill. As a result, the residential area was divided into fishing and farming 


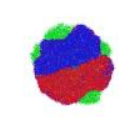

(1) Stage 1

(2) Stage2

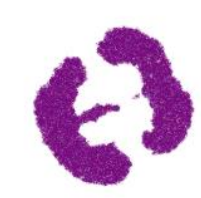

(3) Stage 3

Fig. 2 Stop growing and disruption of villages

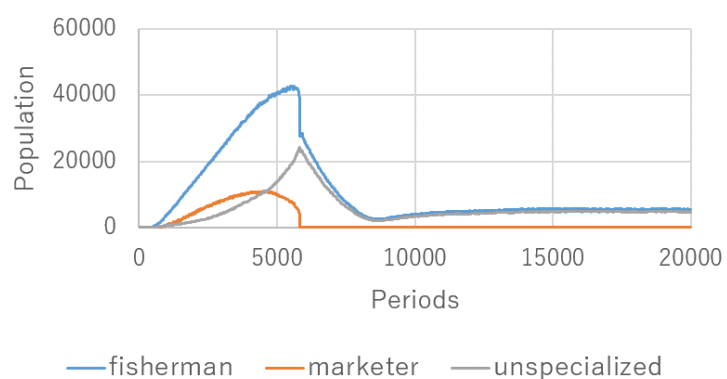

Fig. 3 Transition of population

villages, each of which was occupied by the actors who had a higher skill for a single kind of tasks [5]. After villages grew beyond a limitedly larger size, specialized marketers appeared at the surrounding area to help fishermen and farmers to exchange their service, since the specialized fishermen and farmers cannot carry out exchange by themselves because of the longer distance lying between the villages.

\section{Motivating Value Cocreation and Localizing}

In the most cases of the previous simulation, the residential area of actors enlarged by the limit and split up into two or three villages, such as fisherman, farmer, and marketer villages, as shown in Fig. 1. Fishermen and farmers individually configured a large half-circle region whose boundary was attached at the diameter line of the half circle, and marketers resided on the both ends of the boundary to help the fishermen and farmers to exchange their service. There were no small colony of fishermen or farmers, and they came together into either of single-rolled big villages. It was strange, as compared with the actual human cities and towns that are dispersedly located. Additionally, sometimes when the size of village went beyond the limit, selfservice actors appeared at the surrounding of the circle, as actors with dark purple color shown in Fig.2 (2). These actors stopped being specialized into a single skill, turned to be unspecialized in both fishing and farming skills, and kept their minimum life levels for survive to collaborate with other unspecialized actors. While growing the residential area at the first stage, the actors had to concentrate on a single-rolled task and tightly collaborate with each other to obtain sufficient nutrition for survive, since the economic conditions were unstable. After saturating their growth, however, the conditions became stable, and they started choosing their self-service lives without relying on marketers. If the self-service actors raised up the number of their members, the marketers lost their roles for exchange and disappeared. If the marketers disappeared, the fishermen and farmers lost partners who helped them to exchange, then they also disappeared. Such negative spiral sometimes led to a sudden death of the whole villages, as shown in Fig.2 (3) and (4). Fig. 3 shows the transition of populations in the same case of Fig.2 for their categories of skill types. The populations of fishermen first increased, and then marketers followed. After a while unspecialized actors appeared, the marketers suddenly disappeared and fishermen decreased the number.

These observations and the assumptions consistent with the observations are very interesting but they are not likely to the real world. We guess there are two reasons to lead to such consequences. One is that the exchange skill permits actors to travel very far per an exchange to connect specialized actors so that specialized actors living on the frontier can be easily connected to the actors specialized to the opposite skill. This was a good thing for the specialized actors, but it prevented the actors to generate localized small communities to exchange their service with tightly coupled relationship. Another is that the necessary amount of fish and wheat for surviving was fixed so that actors once satisfying this minimum condition have no motivation to gain more values to make their lives better. As a result, they did not spend their lives for serving more valuable things, but tended to reduce their risks of relying on the marketers and change to serving their values by themselves.

According to such examination, we extended our simulation systems with two options. The first option is changing an amount of sufficient nutrition to be 
variable. We define the minimum required amount of nutrition, which is the same as the existing fixed amount, and make a new rule for giving extended lives to the actors who obtain more nutrition beyond the required amount, as their values. We expect the actors to motivate generating more values without stopping their growth in order to make their lives extended. The second option is a constraints of a shorter trip distance for one-time exchange The specialized marketers can visit many actors to help them to exchange their service, and then the total trip distance may be larger if the marketers have higher skills for exchange. However, since one trip distance is constrained in a shorter distance, they cannot connect actors who live far from each other. This option enhances actors to exchange their service with neighborhood, and then makes more chances to generate small communities in local.

\section{Relational Distance in the Map}

In section 3 , we introduced the localization option, which restricted a trip distance for an exchange in a shorter distance. This seems the opposite direction against the current network trading, where two actors can easily exchange their valuable services even when they live far from each other.

A distance in a map is usually interpreted as a geographical distance, but we can give alternative interpretation, such as a relational distance between actors. If an actor has a tight relationship with another actor, these actors are allocated close to each other even if their geographical locations are very far. For example, $\mathrm{x}$-axis of the map shows actor's interests about ingesting protein. The left-side stands for vegetarians and vegetable farmers, the center for sea food lovers and fishermen, and the right-side for meat lovers and ranchers. Y-axis shows those about ingesting carbohydrate. The bottom-side stands for rice lovers and rice farmers, the center for wheat lovers and wheat farmers, and the top-side for potato lovers and potato farmers. Fish marketers are locating on the center of $\mathrm{x}$-axis, and connect see food lovers and fishermen. The fish market may be divided into smaller markets for actors with specialize interests. Some fishermen and fish marketers may be more specialized in tuna fish, and then they exchange their service at the very small region in the map. In this example, the location indicates a position of interests, and the distance indicates a conceptual distance between their interests. After the pervasion of the internet, we can find a lot of niche markets in the network. If the niche market is very small and weak, a single large market covers all of the niche areas. On the other hand, if

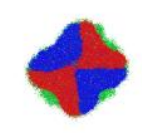

(1) Stage 1

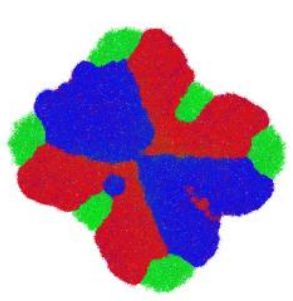

(3) Stage 3

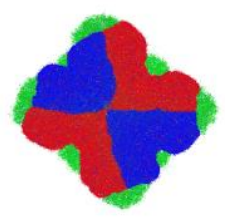

(2) Stage2

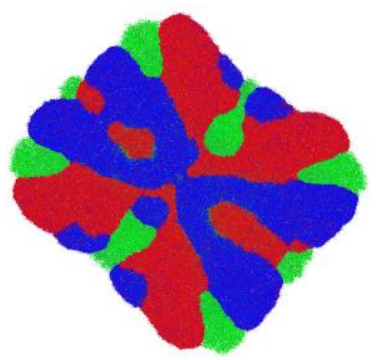

(4) Stage 4

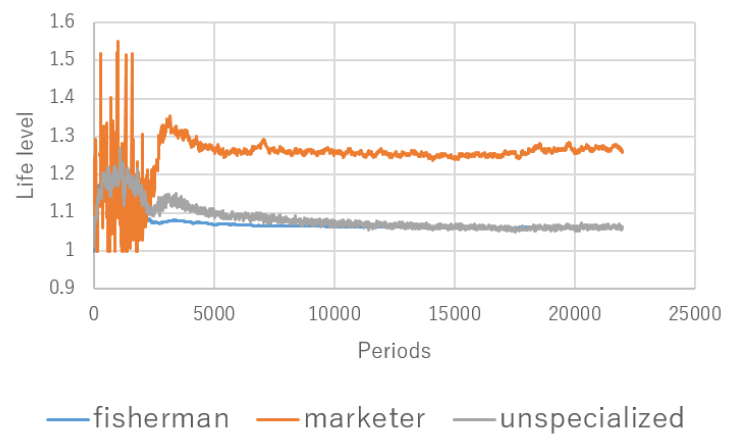

Fig. 5 Transition of life levels

niche markets grow beyond a trading volumes sufficient enough to keep their independent markets, many small niche regions dispersed in the map.

When a travel distance is not restricted, marketers tend to be global marketers, and when the distance is restricted, marketers tend to be niche marketers. We can control and observe the various types of service markets in the simulation.

\section{Simulations}

\subsection{Restricted Trip and Variable Life Levels}

In the first simulation, we applied the restricted trip distance and variable amounts of sufficient nutrition for getting higher life levels. Fig. 4 shows a sample of simulation results. As compared with Figures 2 and 3, villages are divided into small areas, where fishermen, 

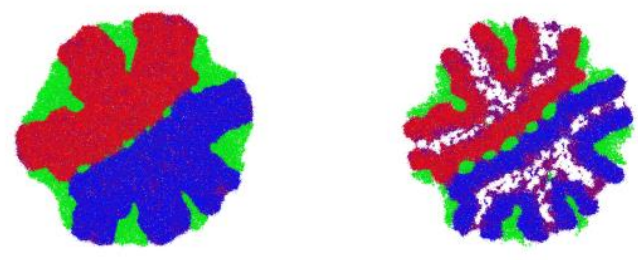

(1) Stage 1

(2) Stage2
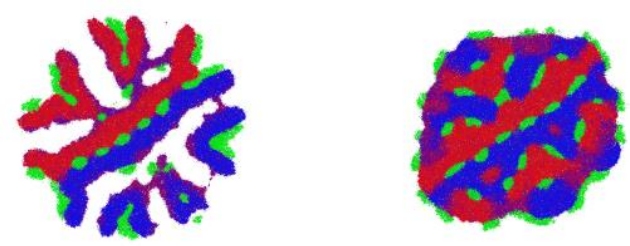

(3) Stage 3 Fig. 6 Macro-micro emergence

farmers and marketers are complicatedly residing and attaching to the others. Marketers, especially, changes their locations from the surrounding areas to the center.

Fig. 5 shows a graph of life levels of the specialized groups. It is remarkable that the life levels of the marketers are much higher than the others. The fishermen and farmers have limitation of their service amounts because natural resources of fish and wheat are restricted. On the other hand, the marketers can serve a lot of service exchange and achieve higher life levels relatively easier than fishermen and farmers. The marketers have no direct restrictions from natural resources, and then their life levels become higher than the others. It's to be noted that some fishermen and farmers create much more values for their lives by collaborating with many actors. Values are not static but determined by the beneficiaries [12].

As viewed in the relational distance, the types of actors' interests become more variety, and the smaller markets appears in corresponding to their individual interests. In order to ingest protein and carbohydrate sufficient enough to survive, separate service markets are configured locally, and actors who prefer to obtain and generate the same types of protein come together and make a local market. Each market may be governed by a different local rule and institution.

\subsection{Macro-Micro Emergence}

As described in the previous research, we applied macro-micro emergence rules to the simulation.
Usually, something in a macro level unexpected emerged by micro interactions among micro entities is defined as emergence [1][2], but sometimes an inverse idea that a macro recognition on micro interactions triggers the next step of micro interactions is also called emergence. Such a macro-to-micro emergence is also important in human lives. In our previous research, an actor optionally calculated the entropy of the types of neighborhood actors, found the most profitable place in the map in correspondence to the entropy, and moved to the place [5]. The experiments in this subsection applied the same rules to actors. In this case, actors are eager to move to better places for surviving, and the markets are likely growing to the matured states quickly. Such rapid growth often leads to partial corruption of the communities to regenerate their inner structures. See Fig. 6 as an example of macro-micro emergence. The community are rapidly generated and localized at stage 1, and the generated local structures were corrupted at stages 2 and 3 . After the corruption, small markets and small communities governed a whole structure of the villages. We must say that the market structures after corruption were not stable, and repeated partial corruption and regeneration at stage 4 . The market continuously kept adapting to the temporal contexts in actors forever.

As viewed in the relational distance, the phenomena occurring in this market seem to be related to a disruptive innovation. At stage 1, a stable big market existed, but their boundaries were expanding to niche and weak regions. After a while the market disrupted from the inside and regenerate a set of brandnew small markets. Before and after the disruption, the institutions governing the market are drastically changed. The total size of markets are similar before and after the disruption, but resolutions and connectivity are much different from the original.

\subsection{Multiple Fertile Lands}

In the real world, fertile regions are scattered over the country, and these regions become cores of local cities or towns. The final setting simulates such scattered fertile regions. Actors are first allocated at the center of circle, as the same condition as the others, and multiple small fertile lands are located around the center in a grid form. The surrounding areas out of the fertile lands are so poor that keeping survive is very severe. When actors move beyond the poor lands, they can find fertile lands again. As a result, residential areas sometimes jump to the next fertile lands, even if few actors live between the fertile lands. We suppose that such scattered fertile lands enhance actors to generate local service ecosystems. 


\section{$\bullet$}

(1) Stage 1

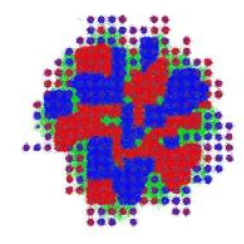

(3) Stage 3 Fig. 7 Multiple fertile locations

Figure 7 shows a sample of multiple fertile lands. At stage 1, additional three small regions appeared as a result of jump from the center. At stages 2 and 3, small regions were connected to grow up to a complex village with marble pattern of fisherman, farmer and marketer regions. After stage 3, small dark purple colored regions of self-service actors appeared around the center village, and finally marketer islands with green color appeared like satellite cities to connect local self-service regions, as shown at stage 4 . These islands may have locally governed rules and institutions different from the center village. Such observations and examinations are very interesting from a viewpoint of S-D logic, because the institutions are endogenously generated [13].

As viewed in the relational distance, the multiple fertile lands seem to be small interest groups with small niche markets. Marketers connect such small niche markets to exchange similar but little different service. The main region at the center is definitely big, but niche small markets also keep their business and lives. It reminds us the current internet market, where big web marketers occupy big shares of markets, but small web markets also keep their business according to focusing on attractive niche groups.

\section{Discussion}

We have examined qualitative explanations of S-D logic with an agent-based simulation. In the previous research papers, we designed an agent model to interpret S-D logic entities into system descriptions [4][5]. For example, we defined collaboration models in which the collaboration with more actors integrated the individual actors' resources to gain more valuable outcomes. We also defined service exchange models in which higher skills for exchange increased their opportunities to complete the service exchange with more partners. These models worked well in a simple simulation, but the configured service market homogeneously grew to generate a single gigantic market even when the size of actors passed beyond 100 thousand.

In this paper, we proposed several ideas to make the simulations closer to the phenomena observed in the real world. First, we restricted a trip distance for one-time exchange. This restriction intended to raise opportunities of the local service exchange to activate localized interactions among actors. This intention was realized in the simulation results where a large village was divided into small regions and marketers mediated the service exchange between the two-sided actors, like fishermen and farmers living close to each other. The result implicates that a complex multidimensional evolution occurs simultaneously in micro, meso and macro contexts in S-D logic [3]. Interactions among actors, such as collaboration and exchange, are so complex processes that local structures are accumulated to build up a total ecosystem.

Second, we gave variable life levels to the actors in accordance with the obtained values. S-D logic defines that service has a quality level, and the values obtained from the service are phenomenologically determined by the beneficiaries [12]. That definition is interpreted as that the value of the service is variable for the actors. In spite of this interpretation, the previous simulations fixed the value levels for all actors. If all actors satisfy the same level of quality of service, they do not try to expand their activities to generate more values, and then they stop growing. The simulation in this paper differentiated the actors' service levels and the required amounts of nutrition. As a result, marketers gathered more nutrition to raise their life levels, and could not stop their growing. S-D logic says that value is also impacted by the sustainability of the social system that enables value creation [3].

Third, we defined another type of fertile map where the fertile lands are scattered over the map in a grid form. As a result, more sophisticated small regions appeared in the simulation. Especially, we could observe satellite cities that were apart from the main market, and local marketers connect small regions to help the actors to exchange their service. These implies unique and various contexts for exchange. In the simulation, many phase transitions were observed, as described by Holling [6]. The transitions occurred not 
only once, but occurred cyclically in the map. This is an important thing described that the service ecosystems are not static nor stable, but dynamically adapted to the situations appeared in the society. S-D logic denotes that there is continuous change in the context, because each actor in the context is always integrating and exchanging resources with other actors and thereby serving other actors [14].

We gave alternative interpretation to the map, where a distance is determined not as a geographical distance, but as a relational proximity among actors. The internet reduced the obstacles of physical distances, and zoomed up niche markets as profitable markets. The standing point of the market is very important to succeed the business. Not only a global market but a new niche market is developed through the internet. Through the simulation, many small groups with small scopes of their interests were generated from a viewpoint of the relational distance.

The main concept of S-D logic is very simple; all economical activities are service, but it can explain emerging complex structures and institutions among actors. Complexity, emergence and self-organization are crucial characteristics of service ecosystems [13]. The complexity is emerged from micro interactions of actors to obtain their own values. The proposed simulation proved a part of the power described in S-D logic, because multiple agent-based simulation worked well and suitable to express and achieve interactions among actors.

\section{Conclusion}

This paper analyzed social phenomena resulted in S-D logic with agent-based simulation. An agent plays a role of an actor in S-D logic, that interacts with the other actors to generate valuable outcome by integrating resources. Especially, this paper focused on localized activities in small interaction communities. In order to achieve such demonstration, the trip distance for one-time exchange was restricted, and the life levels were differentiated according to their quality of service. We also changed the land model into that with multiple fertile lands, and observed small satellite villages appeared apart from the main market. We gave alternative interpretation to the map, where a distance was interpreted as a relational proximity among actors. This interpretation is suitable to explain the market in the internet. In future, we keep improving this simulation and explaining the concept of S-D logic quantitatively and more in detail. We also need statistical analysis of the phenomena. There are a variety of phenomena observed in the simulations, but some are categorized into a small set of patterns. We need to analyze and reorganize them, and extract important parameters to affect the results.

\section{References}

[1] M. Bedau, "Weak emergence," Philosophical Perspectives: Mind, Causation, and World, 11:375--399, 1997.

[2] M. Bedau, "Downward causation and the autonomy of weak emergence," Principia, 6(1) 5--50, 2002.

[3] J. D. Chandler and S. L. Vargo, "Contextualization and value-in-context: How context frames exchange," Marketing Theory, vol. 11, no. 1, pp. 35-49, 2011.

[4] S. Fujita, C. Vaughan and S. L. Vargo, "Service ecosystem emergence from primitive actors in service dominant logic: An exploratory simulation study," Hawaii International Conference on System Sciences, pp. 1601-1610, 2018.

[5] S. Fujita, C. Vaughan and S. L. Vargo, "Service Ecosystems Emergence and Interaction: A Simulation Study," Hawaii International Conference on System Sciences, 2019.

[6] C. S. Holling, "Understanding the Complexity of Economic, Ecological, and Social Systems", Ecosystems, Vol.4, pp. 390-405, 2001.

[7] R. F. Lusch and S. L. Vargo, "The Service-dominant Logic of Marketing: Dialog, Debate, and Directions". Sharpe, 2006.

[8] R. F. Lusch and S. L. Vargo, "The Service-dominant Logic of Marketing: Dialog, Debate, and Directions". Sharpe, 2006.

[9] S. L. Vargo and R. F. Lusch, "Evolving to a new dominant logic for marketing," Journal of Marketing, vol. 68, no. 1, pp. 2-17, 2004.

[10] S. L. Vargo and R. F. Lusch, "Service-dominant logic: Continuing the evolution," Journal of the Academy of Marketing Science, no. 36, pp. 1-10, 2008.

[11] S. L. Vargo, H. Wieland and M. A. Akaka, "Innovation through institutionalization: A service ecosystems perspective," Industrial Marketing Management, vol. 44, pp. 63-72, 2015.

[12] S. L. Vargo and R. F. Lusch, "Institutions and axioms: an extension and update of service-dominant logic," Journal of the Academy of Marketing Science, vol. 44, no. 1, pp. 523,2016

[13] S. L. Vargo and R. F. Lusch, "Service-dominant logic in 2025," International Journal of Research in Marketing, vol. 34, no. 1, pp. 46-67, 2017. 
[14] S. L. Vargo, M. A. Akaka, and C. M. Vaughan,

"Conceptualizing Value: A Service-ecosystem View," Journal of Creating Value, 2017. 